\title{
Mapeamento Sistemático sobre o desenvolvimento e a utilização de Jogos e Simuladores no ensino de Redes de Computadores
}

\author{
Garibaldi da Silveira Júnior - IFFAR - garibaldi.dsj@gmail.com \\ Roseclea D. Medina - UFSM - roseclea.medina@ gmail.com \\ Paulo R Barbieri Dutra Lima - IFFAR - paulo.lima@iffarroupilha.edu.br \\ Alex Eder da Rocha Mazzuco - IFFAR - alexmazzuco@gmail.com
}

\begin{abstract}
Resumo. As disciplinas que abrangem o conhecimento de redes de computadores, presentes na maioria dos cursos técnicos e superiores da área de informática, tendem a possuir uma abordagem teórica na maior parte do tempo, visto que o alto custo dos equipamentos de rede muitas vezes impossibilita a prática. Jogos e simuladores podem representar uma alternativa viável para o ensino destes conteúdos, onde práticas reais podem ser feitas diretamente no ambiente virtual simulado. Este trabalho teve como objetivo analisar como estão sendo desenvolvidos e utilizados jogos e simuladores para $o$ ensino de redes de computadores. O processo foi realizado no formato de um mapeamento sistemático, que resultou no total de 27 artigos. onde foi verificado quais tecnologias foram utilizadas no desenvolvimento dos mesmos, para qual plataforma eles foram desenvolvidos, e quais conteúdos educacionais são utilizados.
\end{abstract}

Palavras-chave. jogos sérios, simuladores, redes de computadores, mapeamento sistemático

\section{Systematic Review of the development and use of Games and Simulators in teaching Computer Networks}

\begin{abstract}
The disciplines that cover the knowledge of computer networks, present in most technical and higher education courses, tend to have a theoretical approach in most of the time, since the high cost of network equipment makes the practice impossible. Games and simulators can represent a viable alternative for teaching these contents, where real practices can be done directly in the virtual simulated environment. This work aimed to analyze how games and simulators are being developed and used to teach computer networks. The process was performed in the format of a systematic mapping, resulting in a total of 27 articles. where it was verified which technologies were used in their development, for which platform they were developed, and what educational content are used.
\end{abstract}

Keywords. serious games, simulators, computer networks, systematic review

\section{Introdução}

O processo de ensino e aprendizagem na sociedade atual requer uma atualização constante por parte da metodologia utilizada pelos docentes, visto que a cada novo V. $17 \mathrm{~N}^{\circ}$ 3, dezembro, 2019 DOI: RENOTE 
artefato de comunicação, surgem possibilidades de utilização dele em um contexto educacional. Esse processo acaba exigindo que o professor habitue-se e passe a conhecer a atual tecnologia, visto que os alunos já estão imbuídos da mesma em seu dia a dia

Quando este assunto é direcionado a um curso da área de informática, podem ser ressaltadas fragilidades da metodologia tradicionalmente aplicada em algumas disciplinas com predominância de conteúdo teórico, como é o caso das que abordam o estudo de redes de computadores. Como dito por Nascimento (2018), os desafios da disciplina acabam por prejudicar a dinâmica do aprendizado, Qian (2017) e Prabawa (2017) citam que para o aprendizado de redes de computadores, é necessário um equilíbrio entre o conteúdo prático e teórico, onde para o estudo efetivo do assunto em questão muitas vezes é necessário o contato com equipamentos de rede, tais como: switches, hubs, controladoras sem fio, roteadores e servidores. Como dito por Santos (2018), tais equipamentos possuem um preço elevado, tornando-se muitas vezes inviável a interação prática do aluno.

Visando resolver o problema citado anteriormente, de acordo com Čabarkapa (2015), foram desenvolvidos simuladores, que são softwares que permitem a configuração de equipamentos virtuais, simulando a conexão de uma rede de forma análoga a utilizada em um ambiente físico, representando uma abordagem de melhor custo benefício para entender os conceitos práticos de instalação e configuração de equipamentos de rede.

A utilização de simuladores como laboratórios virtuais de redes de computadores já é bastante difundida em cursos de informática, Čabarkapa (2015) cita o uso software Cisco Packet Tracer como um dos principais para este fim. No entanto, por tratar-se de software comercial, a licença do mesmo envolve custos, impossibilitando a sua utilização por instituições de ensino com pouco recurso disponível.

A partir disso, novas estratégias foram desenvolvidas, utilizando diferentes abordagens no processo de aprendizado com o uso de simuladores, além da criação de jogos sérios para o propósito de aprendizado da disciplina. Com isso, o objetivo deste trabalho é investigar como está sendo feito o desenvolvimento e o uso de simuladores e jogos no ensino de redes de computadores. Este processo foi realizado no formato de um mapeamento sistemático, sendo feita a análise dos artigos publicados nos últimos cinco anos que abordam tanto o desenvolvimento de um jogo ou simulador como o uso dos mesmos. A pesquisa busca a utilização de tecnologias atuais na metodologia utilizada, sendo excluídas da seleção aquelas tradicionais e com o uso de softwares comerciais. 


\section{Mapeamento Sistemático}

O modelo de pesquisa desenvolvido neste trabalho segue como descrito por Kitchenham (2004), sendo um processo de mapeamento sistemático, o qual é dividido em três etapas: planejamento, condução e discussão dos resultados.

$\mathrm{Na}$ etapa de planejamento, após a definição do escopo da pesquisa, foram criadas as questões norteadoras, que definiram o caminho da mesma. As questões foram definidas de forma a englobar aqueles artigos que relatam tanto o desenvolvimento como uso de jogos e simuladores para o ensino de redes de computadores, sendo elas citadas a seguir:

Q1 - Que tecnologias fazem parte do processo de desenvolvimento e utilização dos jogos e simuladores para o ensino de redes de computadores?

Q2 - Para que plataforma eles são desenvolvidos?

Q3 - Que conteúdos educacionais estão presentes nos jogos e simuladores desenvolvidos?

A partir desta definição, foi determinado o processo de busca dos estudos, que teve como base o trabalho de Battistella e von Wangenheim (2016). Para a busca, foi utilizado o motor de pesquisa de trabalhos acadêmicos Google Scholar, visto que a pesquisa pelo mesmo engloba as principais bases de dados da área de tecnologias educacionais, como IEEE, Springer e ACM, além de apresentar estudos de eventos e revistas nacionais e internacionais. Para otimizar os resultados, foi utilizada a seguinte string de busca, englobando tanto estudos na língua inglesa como em português, e que estivessem publicados entre os anos de 2014 e 2019:

("education" OR "educational" OR "learning" OR "serious" OR "educacional" OR "sério" OR "aprendizado") AND ("game" OR "jogo" OR "simulator" OR "simulador") AND ("Computer networks" OR "redes de computadores")

Esse processo de busca inicial obteve como resultado aproximadamente 14.800 artigos, no entanto, como no estudo citado anteriormente, neste trabalho foram analisados os resultados dos primeiros 300 artigos dispostos pelo motor de busca, visto que os mesmos estão ordenados por relevância, sendo que ela diminuiu drasticamente após os primeiros 150 artigos.

Desta forma, foi dado início a etapa de condução do mapeamento sistemático, onde foram analisados os títulos, resumos e palavras-chave da primeira seleção de artigos. Nesse processo, foram utilizados critérios de inclusão e exclusão para que os mesmos fossem selecionados para a segunda etapa do mapeamento, sendo estes critérios citados na tabela 1 .

Tabela 1 - Critérios de Inclusão e Exclusão 


\begin{tabular}{|c|c|c|}
\hline & Critério de Inclusão (CI) & Critério de Exclusão (CE) \\
\hline 1 & $\begin{array}{l}\text { O estudo relata o desenvolvimento de um jogo ou } \\
\text { simulador para a disciplina de redes de } \\
\text { computadores. }\end{array}$ & $\begin{array}{l}\text { O estudo relata abordagens de ensino tradicionais } \\
\text { com softwares comerciais amplamente utilizados. }\end{array}$ \\
\hline 2 & $\begin{array}{l}\text { O estudo relata o uso de um jogo ou simulador } \\
\text { para a disciplina de redes de computadores. }\end{array}$ & $\begin{array}{l}\text { O estudo mostra apenas um viés teórico ou } \\
\text { representa uma pesquisa de estado da arte ou } \\
\text { mapeamento sistemático. }\end{array}$ \\
\hline 3 & $\begin{array}{l}\text { O estudo encontra-se escrito em inglês ou em } \\
\text { português. }\end{array}$ & O estudo não apresenta resultados claros \\
\hline 4 & $\begin{array}{l}\text { O estudo foi publicado entre os anos de } 2014 \text { e } \\
2019 .\end{array}$ & $\begin{array}{l}\text { O estudo é escrito em outra língua que não inglês ou } \\
\text { português. }\end{array}$ \\
\hline
\end{tabular}

Após a aplicação dos critérios descritos sob os primeiros artigos selecionados, o quantitativo diminuiu para 45 estudos. Estes foram lidos em sua íntegra, buscando analisar por completo se ele atingia algum dos CI. Por fim, após essa segunda análise, foram selecionados 27 artigos, que contemplam a lista final desta pesquisa. A lista dos artigos selecionados após a segunda fase de seleção podem ser acessados pelo link ${ }^{1}$, estando organizados por CI e CE.

\section{Análise dos resultados}

Nesta seção serão discutidos os resultados da pesquisa, que foram classificados de acordo com as questões norteadoras do trabalho, onde foram analisados e organizados pontos específicos de cada artigo.

\subsection{Q1 - Quais tecnologias são envolvidas no processo de desenvolvimento e utilização dos jogos e simuladores para o ensino de redes de computadores?}

Esta questão teve como base analisar como está sendo realizado o processo de criação e utilização dos jogos sérios e simuladores citados nos estudos selecionados para essa etapa. Como pode ser visto na Figura 1, foi verificado o desenvolvimento de 15 jogos, 4 abordagens educacionais utilizando um jogo, a criação de 7 simuladores e 1 tipo de experiência de uso com simuladores não comerciais.

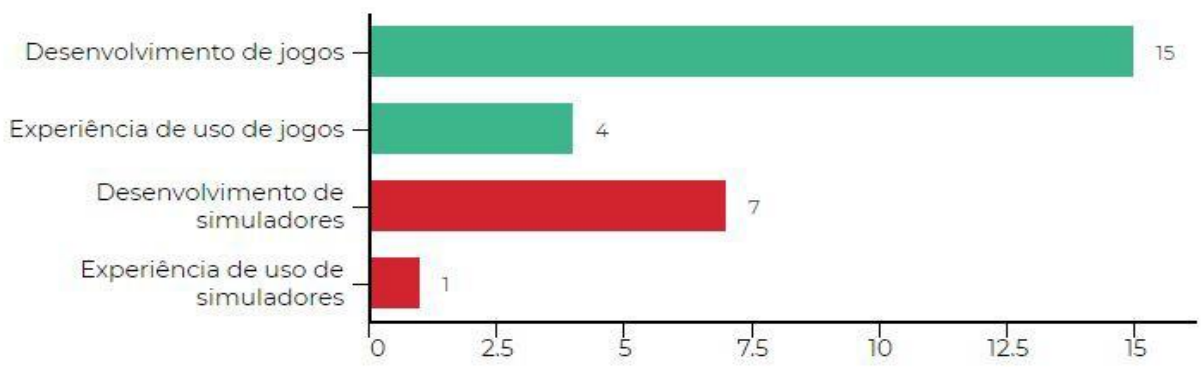

Figura 1 - Desenvolvimento e experiência de uso

${ }^{1}$ https://docs.google.com/document/d/106-aDyxA5VzXfdEnkKdUefwXQdJ O1ag nGCcIDQFYEQ

V. $17 \mathrm{~N}^{\mathrm{o}}$ 3, dezembro, 2019

DOI: 
Pode ser verificado uma incidência maior de desenvolvimento de jogos quando comparado ao de simuladores, tal fato pode ser devido a já consolidação de simuladores comerciais com funções necessárias para o aprendizado, visto que os simuladores desenvolvidos na análise são específicos a um conteúdo. Isso pode ser visto no trabalho de de Souza e Damasceno (2014), onde o simulador criado é específico para a instalação e manutenção de redes, abordando desde a confecção de um cabo de rede a conexão do mesmo em um switch.

Em relação a experiência de uso, também pode ser visto uma incidência maior por parte dos jogos, visto as possibilidades de interatividade que os jogos pode trazer. Isso pode ser visto no estudo de Santos (2016), que utiliza o jogo de computador Counter Strike como uma plataforma de aprendizado, onde o professor criou desafios através de bugs na conexão entre o servidor e os hosts do jogo, onde os alunos precisavam utilizar do conhecimento em redes de computadores para investigar e resolver os problemas de conexão, para poder iniciar o jogo.

Também foram analisados estudos de experiências de utilização de mundos virtuais, como pode ser visto nos trabalhos de Herpich (2014) e Krasmann (2015), onde ambos utilizaram o mundo virtual Cyberciege em uma abordagem para o ensino de redes de computadores.

$\mathrm{Na}$ figura 2, podem ser visualizadas as tecnologias envolvidas na criação dos jogos e experiências de uso analisadas neste estudo. Dentre os artigos selecionados, sete deles não especificaram a plataforma utilizada no desenvolvimento do seu projeto.

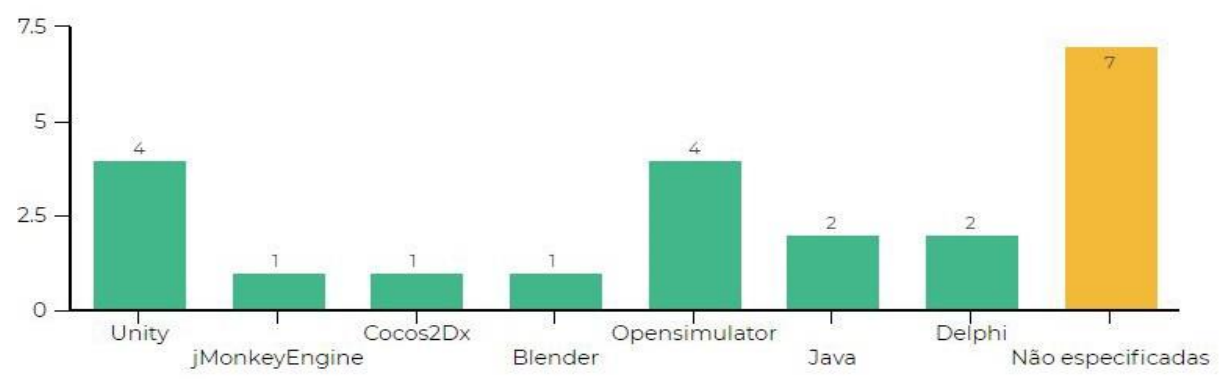

Figura 2 - Tecnologias utilizadas para o desenvolvimento

Foram analisadas quatro pesquisas que utilizam a engine de jogos Unity no desenvolvimento dos jogos, como é o caso de Silva (2019), que desenvolveu um jogo para o aprendizado de protocolos de roteamento, e Jin (2018), que criou um jogo no formato tower defense para o aprendizado de segurança em redes de computadores. $\mathrm{O}$ trabalho de Gramajo (2019), utilizou a engine jmonkeyengine para o desenvolvimento de um jogo em realidade aumentada para o aprendizado de arquitetura de redes, sendo usada a plataforma VUFORIA para integrar os objetos de realidade aumentada ao jogo.

Além disso, outros dois trabalhos utilizaram engines de jogos no seu desenvolvimento, como visto em Nascimento (2018) e Narváez (2014), onde o primeiro 
usou a plataforma de criação de jogos Cocos $2 \mathrm{Dx}$, e o segundo estudo relatou o desenvolvimento de um jogo de de cartas usando a engine de games do software Blender. Ambos os estudos utilizaram como conteúdo educacional o conhecimento relativo a pilha de protocolos TCP/IP.

Também foi percebido um número relevante de artigos que abordaram o desenvolvimento e a utilização de mundos virtuais para o processo de ensino e aprendizagem dos conteúdos de redes. Quatro estudos utilizaram o Opensimulator para criar mundos virtuais, como é o caso de Krasmann (2016), que desenvolveu o jogo ubíquo Jaspion utilizando a plataforma, e de Voss (2014), que criou o TCN5, que é integrado ao ambiente virtual de aprendizagem (AVA) Moodle, e permite que atividades educacionais possam ser resolvidas dentro do mundo virtual.

Quanto ao desenvolvimento de simuladores, em dois trabalhos foi utilizado a linguagem Java para a criação do mesmo, como pode ser visto em Lourenço (2018) que criou um simulador para auxiliar no aprendizado de algoritmos de roteamento para otimização de redes. Além disso, foram encontrados dois trabalhos utilizando a linguagem Delphi, onde Alshawwa (2019) utilizam a mesma para a criação de uma plataforma inteligente para atividades de ensino, dedicada ao treinamento para provas de certificações relacionadas a redes de computadores.

\subsection{Q2 - Para qual plataforma eles são desenvolvidos?}

Esta questão aborda verificar para quais dispositivos os jogos e simuladores foram desenvolvidos. Também foram classificados aqueles estudos que utilizaram de sensores específicos para o seu funcionamento. Na figura 3 podem ser observadas para quais plataformas foram desenvolvidos os jogos e simuladores nos trabalhos avaliados.

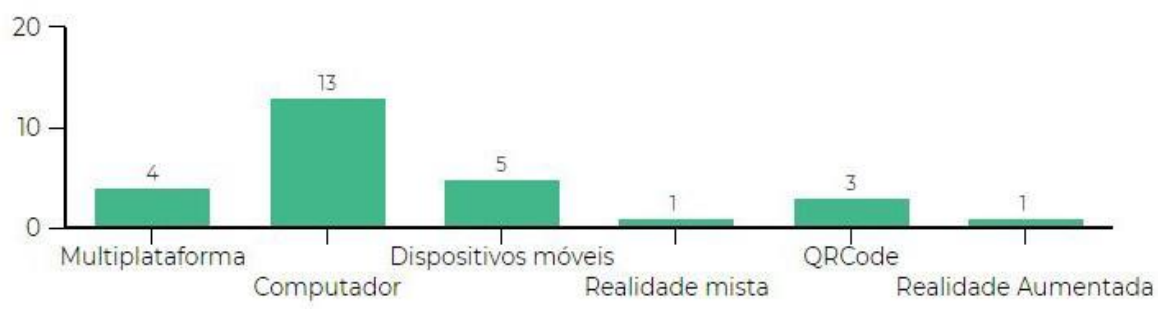

Figura 3 - Plataformas

Como pode ser observado, quatro jogos foram desenvolvidos no formato multiplataforma, como é o caso de Silva (2019) e Nascimento (2018), sendo o primeiro desenvolvido com a engine Unity e o segundo com a Cocos2Dx. Dentre aqueles que utilizaram o computador como plataforma, cinco estudos eram relacionados a mundos virtuais, quatro eram simuladores desenvolvidos com linguagens de programação tradicionais, como Java e Delphi e os outros quatro representavam abordagens utilizando o computador como instrumento, como é o caso de Prabawa (2017), que utilizou o software Wireshark com uma abordagem no estilo de jogo capture a bandeira, 
onde os mesmos deveriam capturar pacotes TCP/IP no software, para cumprir determinados objetivos.

Dentre aqueles desenvolvidos para dispositivos móveis, pode ser percebido que três deles não especificaram a tecnologia utilizada para o desenvolvimento, como visto em Alotaibi (2018) e Švábenský (2018), onde ambos produziram jogos para o sistema android para o aprendizado de segurança de redes.

O trabalho de De Raffaele (2016) utilizou realidade mista em uma proposta de simulador de arquitetura de redes. Onde objetos tangíveis, que representavam os elementos de rede, eram posicionados em uma mesa. O sistema era projetado na mesma com o auxílio de um projetor, e a interação com os objetos era feita via software.

Três trabalhos utilizaram QRCodes na abordagem, como é o caso de Katsadouros (2017) e Borrego (2017), onde o primeiro utiliza um formato de caça ao tesouro para o aprendizado de segurança de redes, onde os jogadores buscaram QRCodes espalhados em determinado local, que deveriam ser escaneados, para gerar uma senha encriptada. $\mathrm{O}$ segundo transformou a sala de aula em uma escape room, onde os alunos utilizaram dos recursos disponíveis, como um laptop e walkie talkies, para resolver problemas com algoritmos de segurança de redes, como o RSA, e encontrar a chave para a saída da sala.

\subsection{Q3 - Quais conteúdos educacionais estão presentes nos jogos e simuladores desenvolvidos?}

Esta questão analisa os conteúdos envolvidos no desenvolvimento dos jogos. Na figura 4 podem ser visto o gráfico que demonstra a quantidade de trabalhos em que determinado conteúdo estava presente. Nesse caso, alguns estudos envolveram mais de um conteúdo, portanto, a soma do quantitativo presente no gráfico supera ao número de estudos avaliados.

Como pode ser visto, boa parte dos trabalhos abordaram o conteúdo de segurança de redes de computadores, pode ser entendido pelo potencial que a disciplina traz ao abordar de assuntos como criptografia e ataques virtuais, onde exemplos de difícil entendimento da forma tradicional, como o conceito de chaves públicas e privadas, podem ser entendidos de uma forma prática, como pode ser visto em Gramajo (2019).

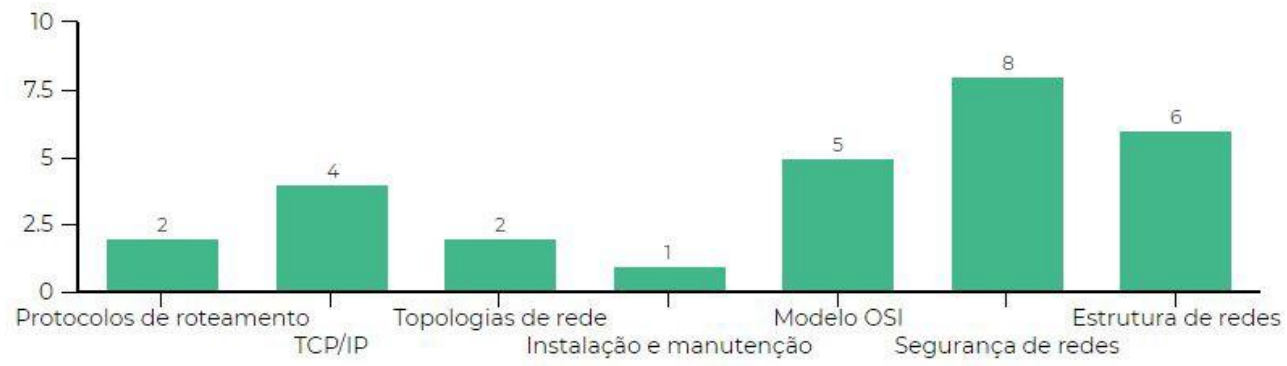

Figura 4 - Conteúdo

V. $17 \mathrm{~N}^{\mathrm{o}}$ 3, dezembro, 2019

DOI: 
buscando trazer os conceitos abstratos que fazem parte desses conteúdos para um formato de jogo, como pode ser visto em Nascimento (2018) e Uiphanhit (2019). Além disso, dois trabalhos abordam protocolos de roteamento, como o de Lourenço (2018) e outros dois trazem conceitos de topologias de rede, como é o caso de Santos (2016).

Usando os conceitos de estrutura de redes, alguns jogos e simuladores permitiram aos jogadores analisar o comportamento de cada equipamento e elemento que faz parte das redes de computadores, como visto em Santos (2018) e De Raffaele (2016). Além disso, trabalhos como De Souza e Damasceno (2014) abordam conceitos técnicos referente a estrutura de redes, sendo relacionado a instalação e manutenção das mesmas.

\section{Conclusão}

O presente mapeamento sistemático permitiu a coleta de dados referentes a atual situação do desenvolvimento e utilização de jogos e simuladores na disciplina de redes de computadores. Neste sentido, foram selecionados 27 estudos, a fim de buscar quais tecnologias são envolvidas no processo de criação dos jogos e simuladores, as plataformas para qual eles foram desenvolvidos e quais os assuntos predominantes.

Este estudo conclui apontando que foram obtidos resultados importantes quanto as tecnologias, onde dentre as engines utilizadas para o desenvolvimento dos jogos e simuladores, a Unity foi a predominante. Da mesma forma, foi percebido que a forte presença de mundos virtuais nas pesquisa,s indica as possibilidades que podem ser conseguidas, como interatividade entre jogadores e conexão com outros ambientes virtuais de aprendizagem.

Em relação às plataformas, foi percebido um predomínio de jogos e simuladores voltados para o computador, sendo poucos trabalhos relacionados a dispositivos móveis, e apenas um que utilizou de realidade aumentada. Considerando isso, pode ser percebido que essa tecnologia foi pouco explorada para o ensino de redes de computadores.

A predominância de estudos para o conteúdo de segurança de redes demonstrou várias possibilidades de jogos utilizando o assunto de criptografia e ataques virtuais, principalmente em relação a atividades em grupo, no entanto, mostrou a atual saturação de pesquisa quanto a esse conteúdo. Por outro lado, conteúdos relacionados a instalação e manutenção de rede, e estrutura de redes mostraram-se pouco explorados, principalmente quanto aos jogos sérios.

Observando as lacunas de pesquisa encontradas neste estudo, foi definido para trabalhos futuros o desenvolvimento de um jogo sério para o ensino de redes de computadores utilizando realidade aumentada. Com isso, é pretendido que o conteúdo seja relativo a estrutura de redes, visto que a simulação dos equipamentos de rede pode V. $17 \mathrm{~N}^{\circ}$ 3, dezembro, 2019 RENOTE DOI: 
significar uma experiência mais próxima da realidade.

\section{Referências}

ALOTAIBI, F., ET AL. Design and Evaluation of Mobile Games for Enhancing Cyber Security Awareness. Journal of Internet Technology and Secured Transactions (JITST), Volume 6, Issue 2, June 2018.

AlShAWWA, I. A., AL-SHAWWA, M. O., \& ABU-NASER, S. S. An Intelligent Tutoring System for Learning Computer Network CCNA. International Journal of Engineering and Information Systems.Vol. 3 Issue 2, Pages: 28-36 . 2019

BATTISTELLA, P., \& VON WANGENHEIM, C. G. Games for teaching computing in higher education-a systematic review. IEEE Technology and Engineering Education, 9(1), 8-30. 2016

BORREGO, C., FERNÁNDEZ, C., BLANES, I., \& ROBLES, S. Room escape at class: Escape games activities to facilitate the motivation and learning in computer science. JOTSE, 7(2), 162-171. 2017

ČABARKAPA, D. Application of Cisco Packet Tracer 6.2 in teaching of advanced computer networks. INFORMATION TECHNOLOGY AND DEVELOPMENT OF EDUCATION ITRO 2015, p. 153, 2015.

DE RAFFAELE, C., SMITH, S., \& GEMIKONAKLI, O. The aptness of Tangible User Interfaces for explaining abstract computer network principles. In 2016 IEEE Frontiers in Education Conference (FIE) (pp. 1-8). IEEE. 2016

DE SOUZA, H. G. P., \& DAMASCENO, E. F. Uma Ferramenta Tridimensional para Apoiar o Ensino de Instalação e Manutenção de Redes de Computadores. In Anais dos Workshops do Congresso Brasileiro de Informática na Educação (Vol. 3, No. 1, p. 524). 2014

GRAMAJO, M. G., LEZCANO, F. T., LOBO, S. G., JUAREZ, G., \& FRAGA, A. L. SIMNET: Simulation-Based Exercises for Computer Network Curriculum Through Gamification and Augmented Reality. In 2018 IEEE World Engineering Education Conference (EDUNINE) (pp. 1-5). IEEE. 2018

HERPICH, F., JARDIM, R. R., DA SILVA, R. F., VOSS, G. B., NUNES, F. B., \& MEDINA, R. D. CYBERCIEGE: Uma abordagem de Jogos Sérios na educação de Redes de Computadores. RENOTE, 12(1). 2014

JIN, G., TU, M., KIM, T. H., HEFFRON, J., \& WHITE, J. Game based cybersecurity training for high school students. In Proceedings of the 49th ACM Technical Symposium on Computer Science Education (pp. 68-73). ACM. 2018

KATSADOUROS, Evangelos et al. Teaching network security through a scavenger hunt game. In: 2017 IEEE Global Engineering Education Conference (EDUCON). IEEE, 2017. p. 1802-1805. 
KITCHENHAM, B. Procedures for performing systematic reviews. Keele, UK, Keele University, 33(2004), 1-26. 2004.

KRASSMANN, A. L., FALCADE, A., DA SILVA, L. E., \& MEDINA, R. D. Serious Games to Computer Networks Learning With CyberCIEGE: A Case Study in Brazilian Higher Education. In XXIII Workshop sobre Educação em Informática (WEI 2015). 2015

KRASSMANN, A. L. . Jogo sério ubíquo integrado a mundo virtual OpenSim para o ensino de redes de computadores (Jaspion). Dissertação de Mestrado. Programa de Pós Graduação em Ciência da Computação - UFSM. 2016

LOURENÇO, W., DE ARAUJO LIMA, S. J., \& ALVES DE ARAÚJO, S.. TASNOP: A tool for teaching algorithms to solve network optimization problems. Computer Applications in Engineering Education, 26(1), 101-110. 2018

NARVÁEZ, L., CHI, V., LÓPEZ, J., MIRANDA, C. Digital educational resource to support the computer networks course.IJCSI International Journal of Computer Science Issues 2014.

NASCIMENTO, Á. O. F., JANUÁRIA, G. C., PRATES, A. A., JUNIOR, R. A. B., NUNES, D. M., LIMA, J. F., \& FROTA, G. L. EducaRedes: Jogo Digital Educacional como Ferramenta de Auxílio no Ensino da Arquitetura TCP/IP. 2018

PRABAWA, H. W., JUNAETI, E., \& PERMANA, Y. Using capture the flag in classroom: Game-based implementation in network security learning. In 2017 rd International Conference on Science in Information Technology (ICSITech) (pp. 690-695). IEEE. 2017

SANTOS, V. D., DOS SANTOS, E. R. S.,BITENCOURT, R. Counter Strike no ensino de Redes de Computadores. SBC - Proceedings of SBGames. 2016

SANTOS, W. USO DE SIMULADORES COMO FERRAMENTA NO ENSINO-APRENDIZAGEM DE REDES DE COMPUTADORES. Projetos $e$ Dissertações em Sistemas de Informação e Gestão do Conhecimento 4.2 , 2018.

SILVA, Helcio. RIPChallenge: Um Jogo Sério para Auxílio ao Aprendizado do Protocolo RIP. In: Anais do XXVII Workshop sobre Educação em Computação. SBC, . p. 503-512. 2019

ŠVÁBENSKÝ, V., VYKOPAL, J., CERMAK, M., \& LAŠTOVIČKA, M.. Enhancing cybersecurity skills by creating serious games. In Proceedings of the 23rd Annual ACM Conference on Innovation and Technology in Computer Science Education (pp. 194-199). ACM.2018

QIAN, Kai, et al. Hands-on learning for computer network security with mobile devices. 2017 26th International Conference on Computer Communication and Networks (ICCCN). IEEE, 2017. 
UIPHANIT, Thanakorn et al. Packet Warriors: An Academic Mobile Action Game for Promoting OSI Model Concepts to Learners. International Journal of Interactive Mobile Technologies, v. 13, n. 6, 2019.

VOSS, G.B. et al. Construção e Análise de um Mundo Virtual 3D para o Ensino e Aprendizagem de Redes de Computadores. In: Brazilian Symposium on Computers in Education (Simpósio Brasileiro de Informática na Educação-SBIE) p. 322.2014 